ARTICLE

\title{
Tissue-specific 5-hydroxymethylcytosine landscape of the human genome
}

\author{
Bo He ${ }^{1,2,10}$, Chao Zhang (10) ${ }^{3,4,10}$, Xiaoxue Zhang1,2,10, Yu Fan ${ }^{5,6,7,10}$, Hu Zeng (1) 1, Jun'e Liu', Haowei Meng (1) 1, \\ Dongsheng Bai ${ }^{1}$, Jinying Peng ${ }^{1}$, Qian Zhang ${ }^{5,6,7,8 凶}$, Wei Tao ${ }^{3 凶} \&$ Chengqi Yi ${ }^{1,2,9 凶}$
}

5-Hydroxymethylcytosine ( $5 \mathrm{hmC}$ ) is an important epigenetic mark that regulates gene expression. Charting the landscape of $5 \mathrm{hmC}$ in human tissues is fundamental to understanding its regulatory functions. Here, we systematically profiled the whole-genome $5 \mathrm{hmC}$ landscape at single-base resolution for 19 types of human tissues. We found that $5 \mathrm{hmC}$ preferentially decorates gene bodies and outperforms gene body $5 \mathrm{mC}$ in reflecting gene expression. Approximately one-third of $5 \mathrm{hmC}$ peaks are tissue-specific differentially-hydroxymethylated regions (tsDhMRs), which are deposited in regions that potentially regulate the expression of nearby tissue-specific functional genes. In addition, tsDhMRs are enriched with tissue-specific transcription factors and may rewire tissue-specific gene expression networks. Moreover, tsDhMRs are associated with single-nucleotide polymorphisms identified by genome-wide association studies and are linked to tissue-specific phenotypes and diseases. Collectively, our results show the tissue-specific $5 \mathrm{hmC}$ landscape of the human genome and demonstrate that $5 \mathrm{hmC}$ serves as a fundamental regulatory element affecting tissue-specific gene expression programs and functions.

\footnotetext{
${ }^{1}$ State Key Laboratory of Protein and Plant Gene Research, School of Life Sciences, Peking University, Beijing, China. ${ }^{2}$ Peking-Tsinghua Center for Life Sciences, Academy for Advanced Interdisciplinary Studies, Peking University, Beijing, China. ${ }^{3}$ Key Laboratory of Cell Proliferation and Differentiation, School of Life Sciences, Peking University, Beijing, China. ${ }^{4}$ Peking University-Tsinghua University-National Institute of Biological Sciences Joint Graduate Program (PTN), Peking University, Beijing, China. ${ }^{5}$ Department of Urology, Peking University First Hospital, Beijing, China. ${ }^{6}$ Institute of Urology, Peking University, Beijing, China. ${ }^{7}$ National Urological Cancer Center, Beijing, China. ${ }^{8}$ Peking University Binhai Hospital, Tianjin, China. ${ }^{9}$ Department of Chemical Biology and Synthetic and Functional Biomolecules Center, College of Chemistry and Molecular Engineering, Peking University, Beijing, China. ${ }^{10}$ These authors contributed

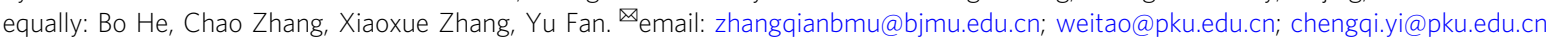


D NA methylation at the fifth position of cytosine $(5 \mathrm{mC})$, which is established and maintained by DNA methyltransferases, is a predominant epigenetic modification that is critical for various biological and pathological processes, including silencing of transposable elements, regulation of gene expression, genomic imprinting and X-chromosome inactivation ${ }^{1,2}$. 5Hydroxymethylcytosine $(5 \mathrm{hmC})$, also known as the "sixth base" of DNA, was discovered as another relatively abundant form of cytosine modification in Purkinje neurons and mouse embryonic stem cells $(\mathrm{mESCs})^{3,4}$. Further studies found that the ten-eleven translocation (TET) family of $\mathrm{Fe}(\mathrm{II})$ - and $\alpha$-ketoglutaratedependent DNA dioxygenases (including TET1, TET2, and TET3) catalyzes the sequential oxidation of $5 \mathrm{mC}$ to $5 \mathrm{hmC}$, 5formylcytosine $(5 \mathrm{fC})$ and 5 -carboxylcytosine $(5 \mathrm{caC})^{4-6}$. Subsequently, the DNA repair enzyme thymine-DNA glycosylase (TDG) can excise $5 \mathrm{fC}$ and $5 \mathrm{caC}$ to generate abasic sites, which eventually results in the regeneration of unmodified cytosines by the base excision repair pathway ${ }^{6-8}$. This TET-TDG pathway is known as the active DNA demethylation pathway.

In addition to being an intermediate of $5 \mathrm{mC}$ oxidation, evidence shows that $5 \mathrm{hmC}$ is a stable epigenetic mark with regulatory functions ${ }^{9}$. The specific genomic distribution pattern of $5 \mathrm{hmC}$, such as its high enrichment in the gene bodies of transcriptionally active genes, promoters and enhancers, hints at specific biological roles of $5 \mathrm{hmC}^{10-12}$. In addition, the $5 \mathrm{hmC}$ level undergoes highly dynamic changes during development, differentiation and cancer ${ }^{4,13,14}$. For instance, the global $5 \mathrm{hmC}$ content is dramatically reduced in multiple human cancers compared with that in the normal tissues adjacent to the cancer ${ }^{15-17}$, suggesting that dysregulation of genomic $5 \mathrm{hmC}$ may be involved in tumorigenesis. Mechanistically, abnormal hydroxymethylation status impacts chromatin structure by interrupting the interaction of $5 \mathrm{hmC}$-specific binding proteins with $5 \mathrm{hmC}^{18}$. Altogether, existing data have suggested a critical role of $5 \mathrm{hmC}$ in developmental processes and an association of dysregulation of $5 \mathrm{hmC}$ with human diseases.
Different methods have been developed to map the genomic distribution of $5 \mathrm{hmC}$, including affinity-based methods $(5 \mathrm{hmC}$ DIP-Seq, CMS-Seq, GLIB, and hMe-Seal) and high-resolution methods (oxBS-Seq, TAB-Seq, hmC-CATCH, TAPS, and ACESeq) ${ }^{19,20}$. For instance, we previously developed $\mathrm{hmC}-\mathrm{CATCH}^{21}$, which is a bisulfite-free method for genome-wide detection of $5 \mathrm{hmC}$. hmC-CATCH couples selective chemical labeling and biotin pulldown ${ }^{21-24}$, thus allowing $5 \mathrm{hmC}$ enrichment and detection at single-base-resolution. While hydroxymethylome maps have been obtained for mammalian cell lines and mouse tissues, $5 \mathrm{hmC}$ profiles in human tissues have not been as comprehensively characterized.

In this study, we generated genome-wide, base-resolution $5 \mathrm{hmC}$ data via hmC-CATCH across 19 human tissue types, represented by 60 tissue samples derived from 6 Chinese donors. We found that $5 \mathrm{hmC}$ is enriched in gene bodies and that it exhibits a better positive correlation with gene expression than gene body $5 \mathrm{mC}$. Tissue-specific differentially hydroxymethylated regions (tsDhMRs) reside in regions enrich for regulatory elements, and co-localize with transcription factor-binding sites, which may regulate the tissue-specific gene expression network. Furthermore, tsDhMRs were shown to enrich disease-related single-nucleotide polymorphisms (SNPs) identified by genomewide association studies (GWAS), linking $5 \mathrm{hmC}$ to human phenotypes and pathologies. Collectively, our results provide a highresolution, high-quality atlas of DNA hydroxymethylation across diverse human tissues and provide a resource for exploring the role of this modification in gene expression networks.

\section{Results}

5-Hydroxymethylcytosine landscape of diverse human tissues. To systematically investigate the dynamics of DNA hydroxymethylation across different human tissues, we collected samples of 19 tissue types from 6 Chinese donors: 3 males and 3 females (Fig. 1a and Supplementary Tables 1 and 2). We profiled
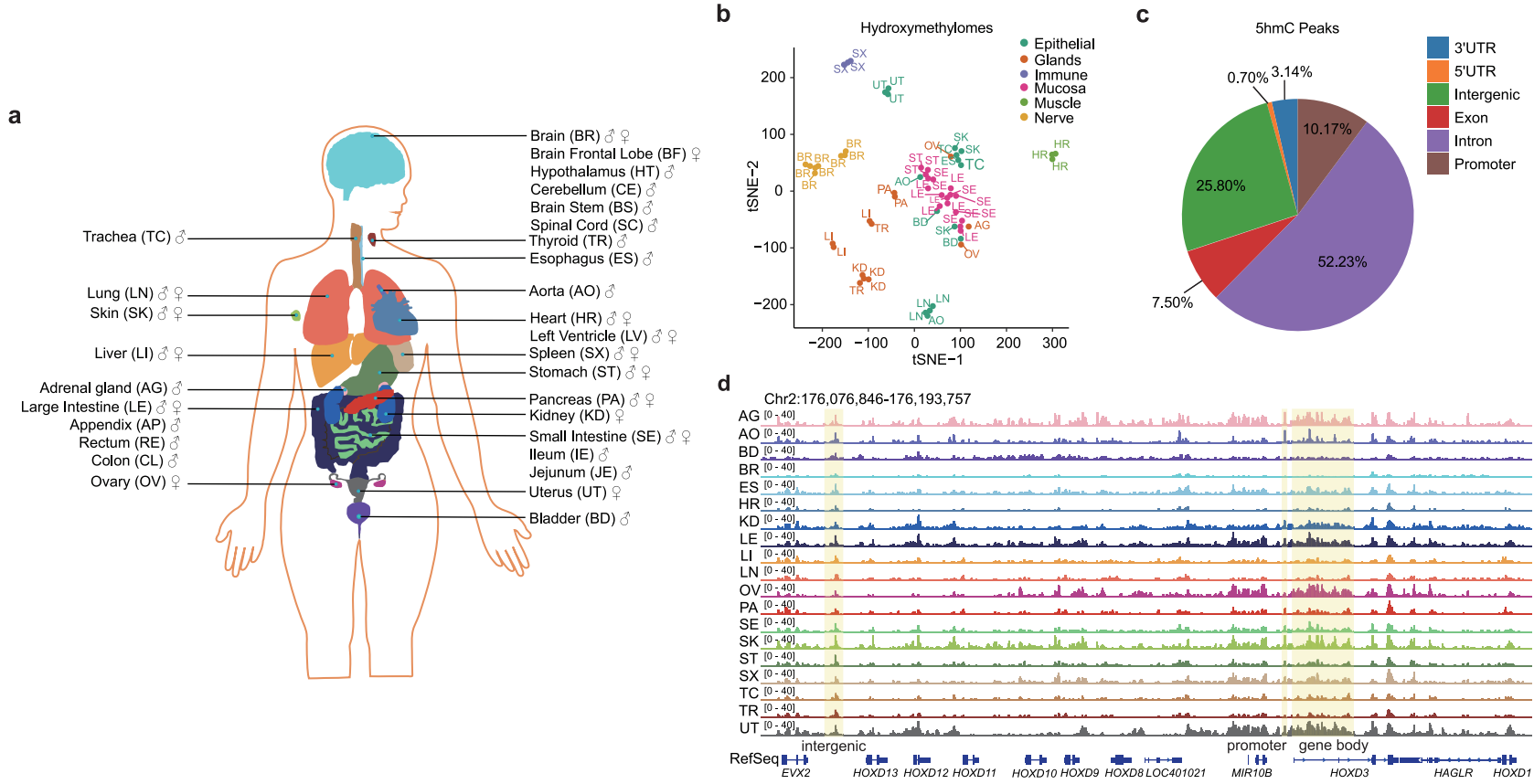

Fig. 1 Hydroxymethylation landscape of human tissues. a Human tissues analyzed in this study. Samples are denoted by the two-letter code in parentheses. Colors indicate each tissue type. $\mathbf{b}$ tSNE cluster of all tissues using the global $5 \mathrm{hmC}$ signals in 10-kb bins. c Pie chart showing the percentage of $5 \mathrm{hmC}$ peaks in each class of genomic features. The promoter regions are defined as $2 \mathrm{~kb}$ around the TSS. d IGV visualization of the $5 \mathrm{hmC}$ signals surrounding the HOXD gene cluster on chromosome 2. $5 \mathrm{hmC}$ signals in promoter, gene body or intergenic region are highlighted. 
the $5 \mathrm{hmC}$ signals of the various tissues via hmC-CATCH $(60$ hmC-CATCH samples; 83-183 million paired-end reads per sample; average, 128 million paired-end reads) (Supplementary Table 3 ). We found that the $5 \mathrm{hmC}$-containing spike-in sequence was specifically and efficiently enriched (Supplementary Fig. 1a) and displayed a high detection rate for $5 \mathrm{hmC}(\sim 91 \%$ and $\sim 98 \%$ before and after pulldown) (Supplementary Fig. 1b, c and Supplementary Table 4), both of which showed the high quality of the hydroxymethylome generated by hmC-CATCH. We found that $\sim 80 \%$ reads in the pulldown samples have C-to- $\mathrm{T}$ conversion and $\sim 94 \%$ of peaks contain at least one confident $5 \mathrm{hmC}$ site; nevertheless, in order to ensure the confidence of detection, we only selected the reads that contain C-to- $\mathrm{T}$ conversion signals (Supplementary Fig. 1d, e). This made sure that all peaks are $5 \mathrm{hmC}-$ enriched regions. It is also worth mentioning that after removing reads without C-to- $\mathrm{T}$ conversion, $\sim 97 \%$ peaks were still identified. In addition, tissues derived from the same organ system from different donors were clearly clustered together (Fig. $1 \mathrm{~b}$ and Supplementary Fig. 1f), further demonstrating the confidence of the $5 \mathrm{hmC}$ data.

We next analyzed the genomic features of $5 \mathrm{hmC}$. We identified 721,404 reproducible $5 \mathrm{hmC}$ peaks, most of which were located in intron and intergenic regions (Fig. 1c). The peak number of each tissue type ranged from 272,391 to 525,080 (Supplementary Fig. 1g). We found that $5 \mathrm{hmC}$ was highly enriched in gene bodies, especially in exon regions, while it was depleted in intergenic regions (Supplementary Fig. 1h). Taking the HOXD gene cluster as an example, we observed $5 \mathrm{hmC}$ signals in distal regulatory elements, promoters and gene bodies; such signals were also dynamic among tissues (Fig. 1d). Hence, via hmC-CATCH, we were able to generate the whole-genome $5 \mathrm{hmC}$ landscape of different human tissues.

Hydroxymethylome at single-base-resolution. To obtain a more detailed and clearer landscape of the hydroxymethylome, we analyzed $5 \mathrm{hmC}$ sites at single-base-resolution. We identified 9,416,937 reproducible $5 \mathrm{hmC}$ sites, with the site number of each tissue type ranging from $1,217,850$ to $2,429,878(\sim 3-10 \%$ of CpG sites were hydroxymethylated) (Supplementary Fig. 2a). While the number of $5 \mathrm{hmC}$ peaks in the brain is comparable to other tissues (Supplementary Fig. 1g, presumably due to the insensitivity of enrichment-based methods to overall modification level change), the number of $5 \mathrm{hmC}$ sites in the brain is significantly higher than that in other tissues $\left(P\right.$-value: $\left.6.57 \times 10^{-7}\right)$, consistent with the higher $5 \mathrm{hmC}$ content in the brain ${ }^{20}$. A representative locus is shown in Supplementary Fig. 2b, in which the single $5 \mathrm{hmC}$ sites identified by hmC-CATCH co-localize nicely with $5 \mathrm{hmC}$ sites by TAB-seq. More than half of the $5 \mathrm{hmC}$ sites were identified in at least two tissues, suggesting that a substantial number of $5 \mathrm{hmC}$ sites could be conserved (Supplementary Fig. 2c). Most of the $5 \mathrm{hmC}$ sites were located in intron and intergenic regions (Fig. 2a) and were highly enriched in gene bodies (Supplementary Fig. 2d).

$5 \mathrm{mC}$ in the CG context is almost symmetrical due to the maintenance of DNA methyltransferase 1 . A total of $98.46 \%$ of the $5 \mathrm{hmC}$ sites identified were located in the CG context (Fig. 2b), but only $13 \%$ of the $5 \mathrm{hmCG}$ sites were symmetrical (Fig. 2c), which is consistent with previous data in $\operatorname{mESCs}^{25}$. Thus, this pattern of $5 \mathrm{hmC}$ differs from that of $5 \mathrm{mC}$, which is symmetrical in human tissues ${ }^{26}$. In addition, the proportion of $5 \mathrm{hmC}$ in the non-CG context $(5 \mathrm{hmCH})$ was variable in different tissues, ranging from $0.96 \%$ to $2.79 \%$ (Fig. $2 \mathrm{~d}$ and Supplementary Fig. $2 \mathrm{e}$ ). Previous research suggests that DNA methylation is rapidly accumulated in $5 \mathrm{mCH}$ sites during synaptogenesis ${ }^{27}$, and we also found a higher $5 \mathrm{hmCH}$ ratio in the brain than that in other tissues (Fig. 2d and Supplementary Fig. 2e), indicating that $5 \mathrm{hmCH}$ may play a role during brain development.

We next analyzed the $5 \mathrm{hmC}$ context in tissues and found a "CA ${ }^{\text {hm }}$ CGT" motif for $5 \mathrm{hmC}$ (Fig. 2e and Supplementary Fig. 2f, g), which coincided with the binding site of ARNT, a housekeeping gene that participates in important metabolic processes. Within ARNT ChIP-seq signals, cytosines are hydroxymethylated in most tissues, with evident C-to-T conversion in the sequencing reads and hence clear single $5 \mathrm{hmC}$ sites (Fig. $2 \mathrm{f}$ and Supplementary Fig. 2h). This observation hints that $5 \mathrm{hmC}$ may positively influence the genomic occupancy of ARNT. Notably, it has been reported that the ARNT binding motif loses $5 \mathrm{hmC}$ signals in esophageal cancer patients compared to healthy individuals ${ }^{28}$. With regard to the $5 \mathrm{hmCH}$ modification, we found that the most frequent base following $5 \mathrm{hmCH}$ was adenine (Supplementary Fig. $2 \mathrm{i}, \mathrm{j}$ ), consistent with the $5 \mathrm{mCH}$ sequence preference ${ }^{26}$.

Taken together, the results of the base-resolution hydroxymethylome analysis presented above revealed the varied $5 \mathrm{hmCH}$ ratio among tissues, the asymmetrical distribution of 5hmCG and the sequence preference of $5 \mathrm{hmC}$, providing a potential mechanism for how $5 \mathrm{hmC}$ can be modified, recognized and dynamically regulated.

Gene body $5 \mathrm{hmC}$ excels $5 \mathrm{mC}$ in reflecting gene expression. As the $5 \mathrm{hmC}$ level in the gene body shows a positive correlation with gene expression in mESCs and the mouse brain ${ }^{18}$, we analyzed whether the gene body $5 \mathrm{hmC}$ level in human tissues also corresponds to gene expression. Indeed, we found that stronger gene body $5 \mathrm{hmC}$ signals were correlated with higher gene expression levels (Fig. 3a and Supplementary Fig. 3a). For instance, the genes that escape $\mathrm{X}$ chromosome inactivation display higher gene body $5 \mathrm{hmC}$ levels than the inactive genes (Supplementary Fig. 3b, c). As the gene body $5 \mathrm{mC}$ level is reported to be positively correlated with transcription ${ }^{1}$, we also compared the gene body $5 \mathrm{mC}$ and $5 \mathrm{hmC}$ levels (Fig. $3 \mathrm{~b}, \mathrm{c}$ ). Although $5 \mathrm{mC}$ co-localized with $5 \mathrm{hmC}$ in gene bodies (Fig. $3 \mathrm{~d}$ ), we found that the gene body $5 \mathrm{hmC}$ level exhibited a stronger quantitative correlation with gene expression levels than the gene body $5 \mathrm{mC}$ level. More specifically, we found $5 \mathrm{hmC}$ is almost linearly positively correlated with gene expression, while $5 \mathrm{mC}$ shows a non-monotonic and bell-shaped relationship with gene expression (Supplementary Fig. $4 \mathrm{a}-\mathrm{e})^{29}$.

To further explore the dynamics of the gene body $5 \mathrm{hmC}$ across human tissues, we defined 4,031 tissue-specifically expressed genes using RNA expression data generated by the GenotypeTissue Expression project $(\mathrm{GTEx})^{30}$ (Supplementary Fig. 4f). The expression of tissue-specific genes and gene body $5 \mathrm{hmC}$ levels also demonstrated a positive correlation, which was absent for $5 \mathrm{mC}$ (Supplementary Fig. 4g-i). Correlations of representative tissue-specific marker genes are shown in Fig. 3e-g. For instance, $P G A 4$ is specifically expressed in the stomach and encodes the precursor of pepsin; we found the highest level of gene body $5 \mathrm{hmC}$ signals in the stomach among all tissues (Fig. 3e, f). In contrast, gene body $5 \mathrm{mC}$ failed to display a positive correlation with tissue-specific gene expression (Fig. 3g). Taking CYP4A11 as another example showed the following: this gene is specifically expressed in the liver and is involved in drug metabolism and the synthesis of cholesterol; consistent with this function, we also found higher gene body $5 \mathrm{hmC}$ levels in the liver than in other tissues (Fig. 3h). Again, this pattern was not found for $5 \mathrm{mC}$ (Supplementary Fig. 4j). As $5 \mathrm{hmC}$ shows a strong co-localization with Poll $^{21}$, it was proposed to play a role in the transcription process. Collectively, these data show that the gene body $5 \mathrm{hmC}$ level correlates well with gene expression and may be associated with the maintenance of tissue-specific functions. 
a

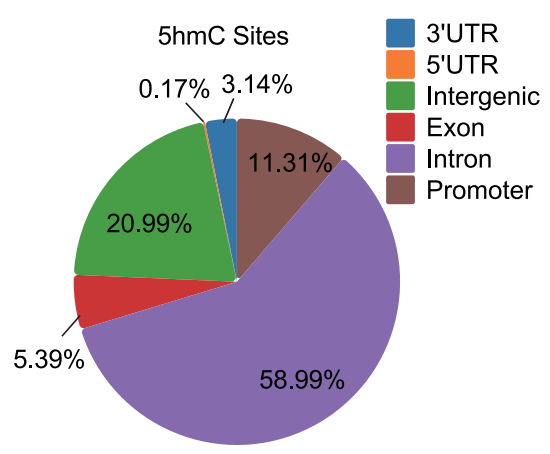

b

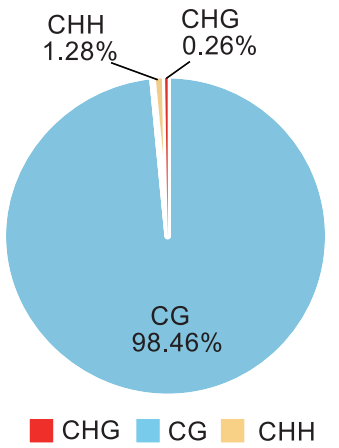

c

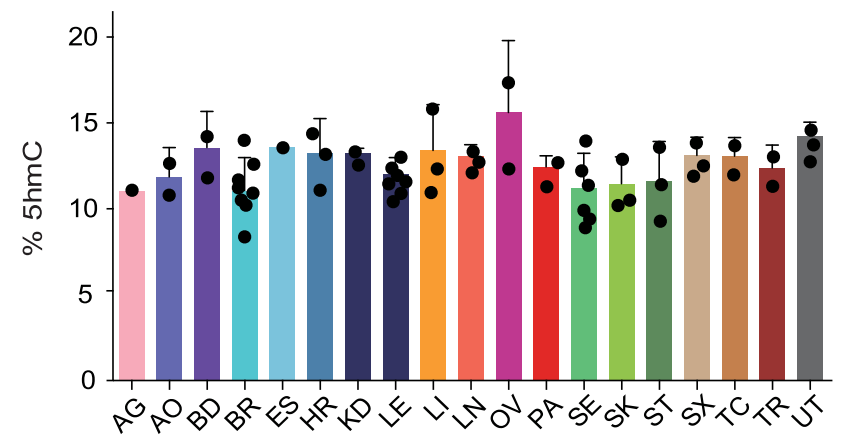

d

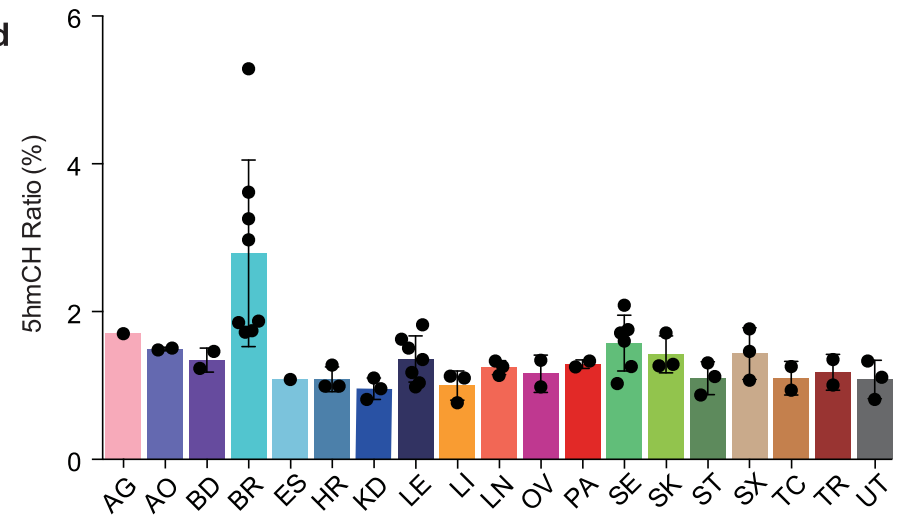

e

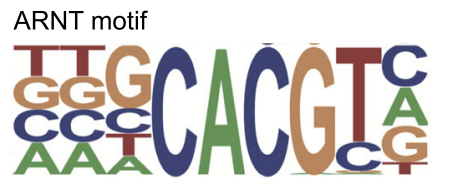

$5 \mathrm{hmC}$ motif in promoter

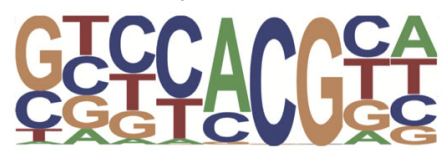

$5 \mathrm{hmC}$ motif in gene body

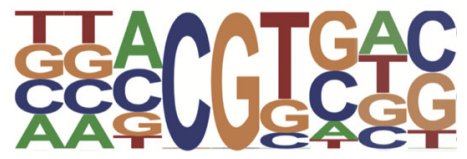

$5 \mathrm{hmC}$ motif in distal region

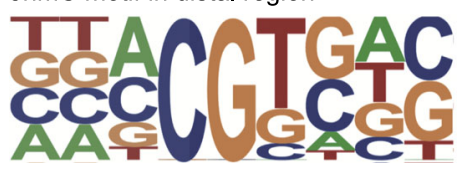

f Chr3:191,355,881-191,356,433

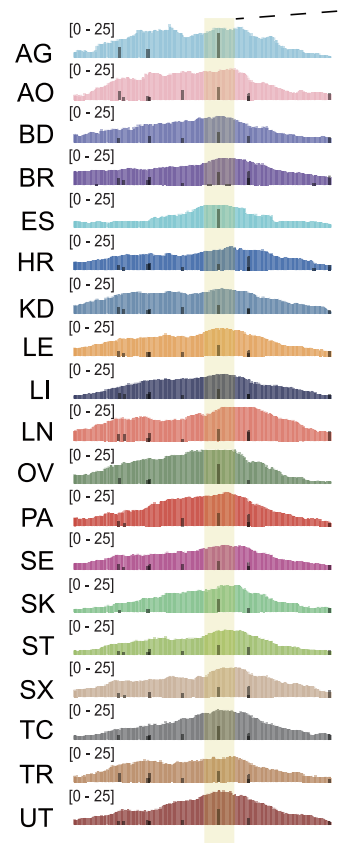

Refseq
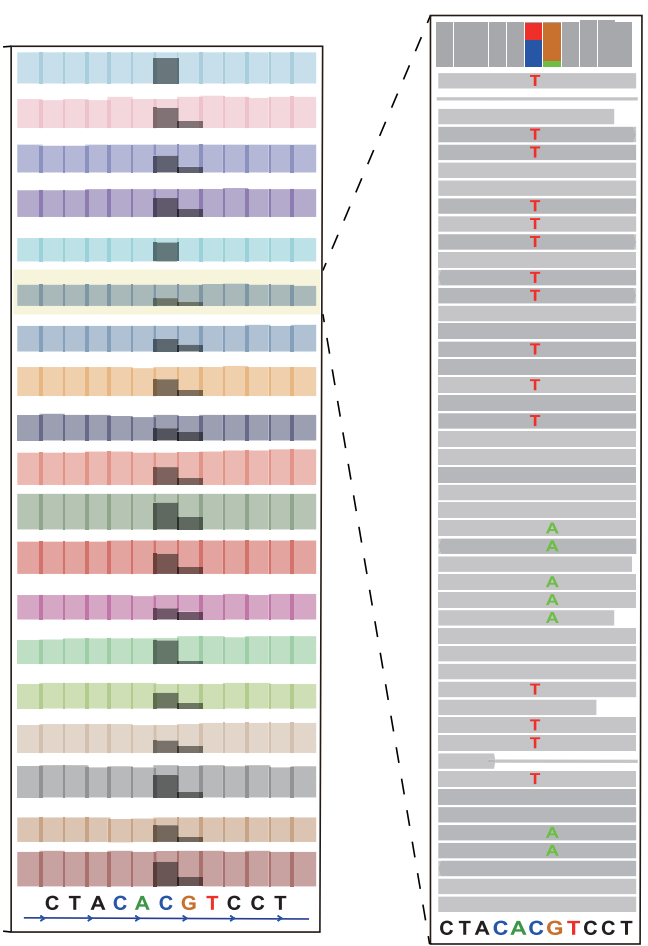

Fig. 2 Single-base-resolution profiles of $\mathbf{5 h m C}$. a Pie chart showing the percentage of $5 \mathrm{hmC}$ sites in each class of genomic features. $\mathbf{b}$ Distribution of $5 \mathrm{hmCG}, 5 \mathrm{hmCHH}$ and $5 \mathrm{hmCHG}$ sites. c Average proportion of symmetrically distributed $5 \mathrm{hmC}$ sites (Data are represented as mean values $+/-\mathrm{SD}$ ). The number of samples are shown in Supplementary Table 2. d Proportions of $5 \mathrm{hmCH}$ in different tissues (Data are represented as mean values $+/-\mathrm{SD}$ ). The number of samples are shown in Supplementary Table 2. e The most significant $5 \mathrm{hmC}$ motif in different genomic elements demonstrates partial sequence overlap with the known transcription factor motif of ARNT. f IGV visualization of the $5 \mathrm{hmC}$ site signals on chromosome 3 . The gray bar represents the Cto- $\mathrm{T}$ counts. $5 \mathrm{hmC}$ is asymmetrical at the $5 \mathrm{hmCG}$ site. 
a

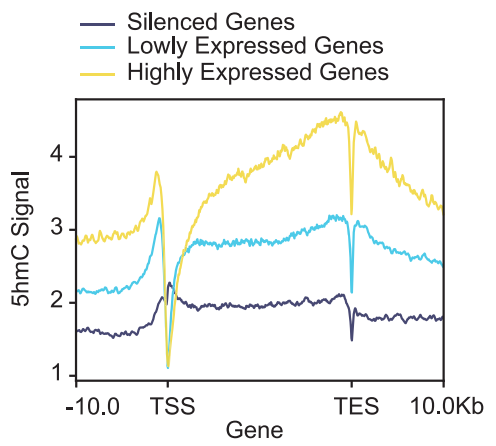

b

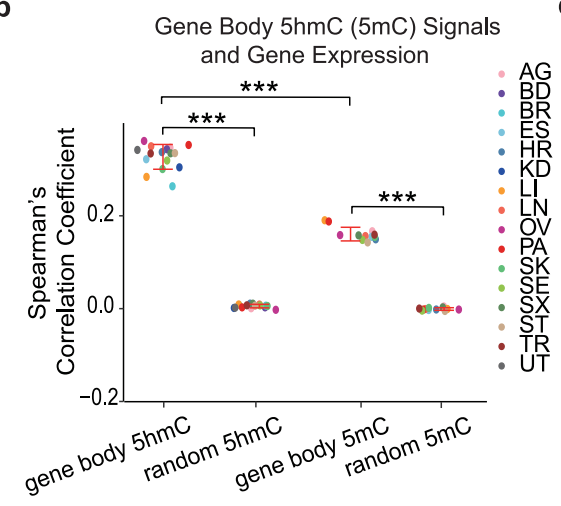

e

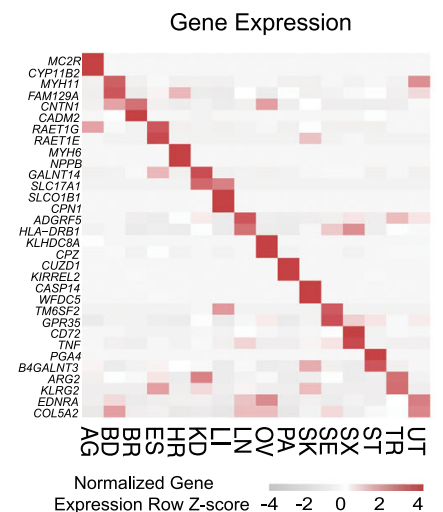

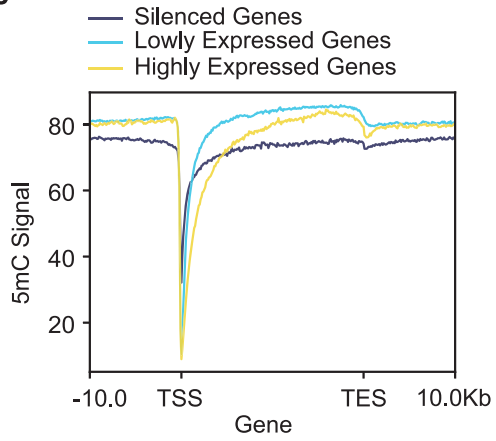

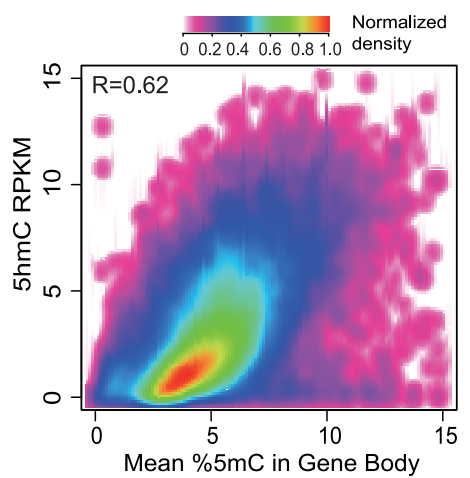

f

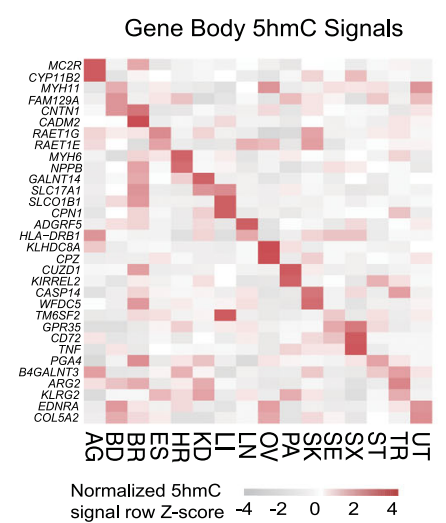

h

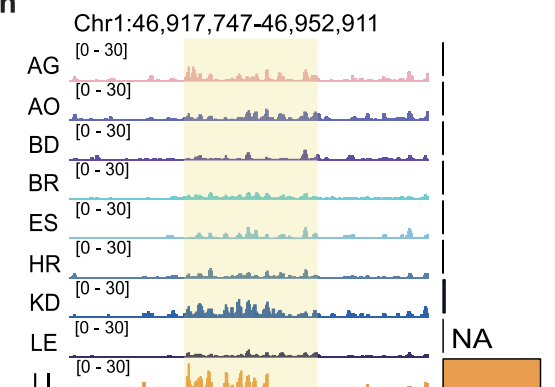

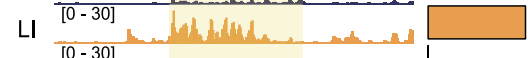

LN ${ }^{[0-30]}$

$V^{[0-30]}$

PA $\frac{[0-30]}{[0-30]}$

SE

ST ${ }^{0-30]}$

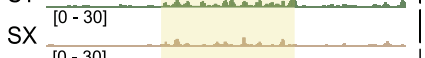

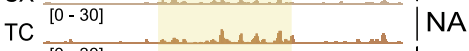

$T R \frac{[0-30]}{[0-30]}$

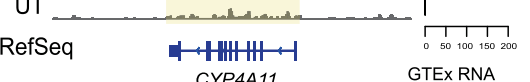

CYP4A11

Expression Level (TPM)

g

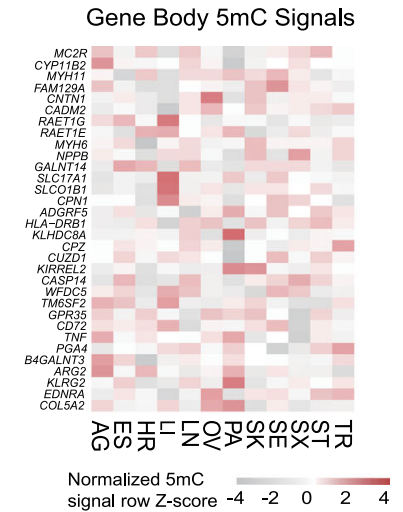

Fig. 3 Gene body $\mathbf{5 h m C}$ correlates well with gene expression in human tissues. a 5hmC profiles of genes expressed at high (yellow), low (cyan), and silenced (blue) levels in heart tissue. b Spearman's correlation of gene body $5 \mathrm{hmC}(5 \mathrm{mC})$ signals and gene expression levels. Random regions were selected as controls ( ${ }^{\star \star *}$ represents $P$-value $<0.0001$; two-side Mann-Whitney $U$-test; $n_{5 \mathrm{hmC}}=16$ tissue types; $n_{5 \mathrm{mC}}=12$ tissue types; $5 \mathrm{hmC}$ vs. random $P$ value: $3.32 \times 10^{-9} ; 5 \mathrm{mC}$ vs. random $P$-value: $7.396 \times 10^{-7} 5 \mathrm{hmC}$ vs. $5 \mathrm{mC} P$-value $: 6.57 \times 10^{-8}$; red lines represent the first and third quantiles). c $5 \mathrm{mC}$ profiles of genes expressed at high (yellow), low (cyan) and silenced (blue) levels in heart tissue. d Scatter plot showing the correlation of $5 \mathrm{mC}$ and $5 \mathrm{hmC}$ signals at gene bodies. e Heatmap displaying the expression levels of tissue-specific marker genes. Gene expression of matched tissue samples downloaded from the GTEx project. $\mathbf{f}, \mathbf{g}$ Heatmap displaying normalized gene body $5 \mathrm{hmC}(\mathbf{f})$ and $5 \mathrm{mC}$ (g) signals of tissue-specific marker genes (order of rows as in $\mathbf{e}$ ). $5 \mathrm{mC}$ data downloaded from ENCODE. $\mathbf{h}$ IGV visualization of the $5 \mathrm{hmC}$ signals at the gene body of CYP4A11 and surrounding regions. Gene expression levels of the gene in different tissues are shown on the right.

tsDhMRs reside in regions that may function as tissue-specific regulatory elements. $5 \mathrm{hmC}$ may participate in development and cell differentiation ${ }^{11,31,32}$; nevertheless, its regulatory functions in different tissues have not been reported. We found $5 \mathrm{hmC}$ showed high enrichment in cis-regulatory elements in the corresponding tissues (Fig. 4a). In contrast, $5 \mathrm{mC}$ are depleted in cis-regulatory elements (Fig. 4b). We identified 33.3\% (240,269 out of 721,404 peaks) of the $5 \mathrm{hmC}$ peaks as being differentially hyperhydroxymethylated in different tissues, which we term as tissue-specific differentially hydroxymethylated regions (tsDhMRs) (Supplementary Fig. 5a, See Methods). Majority of the tsDhMRs are located in intron and distal regions $(56.0 \%$ and $31.5 \%$, respectively, Supplementary Fig. 5b). We further analyzed tsDhMRs at distal regions and found that they are enriched for $\mathrm{H} 3 \mathrm{~K} 4 \mathrm{mel}$ and $\mathrm{H} 3 \mathrm{~K} 27 \mathrm{ac}$ in corresponding tissues, which are putative enhancer marks (Fig. 4c, d and Supplementary Fig. 5c). Thus, tsDhMRs reside in regions with regulatory potential. In addition, tsDhMRs show higher evolutionary conservation than random regions (Supplementary Fig. 5d), suggesting the importance of such functional elements.

We then investigated how these tsDhMRs may contribute to the gene expression programs of specific tissues. We adopted an existing method to identify linkages between tsDhMRs and putative target genes occurring within a $500-\mathrm{kb}$ window ${ }^{33}$. In 
a
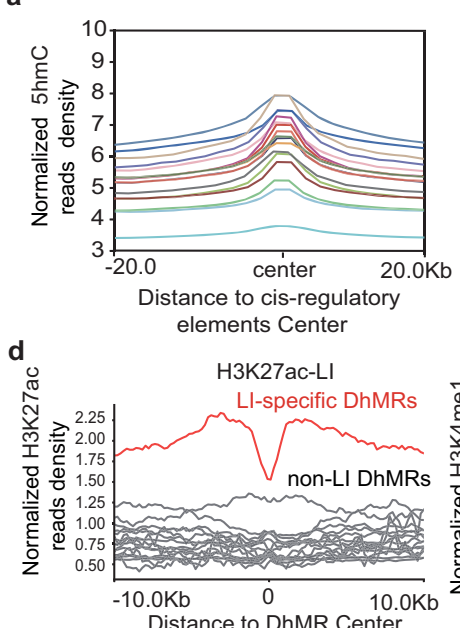

Distance to DhMR Center

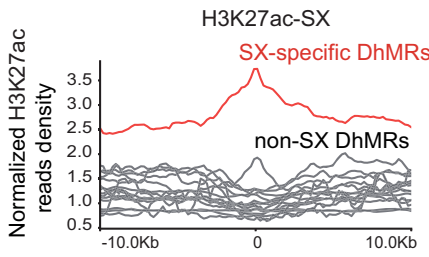

Distance to DhMR Center b

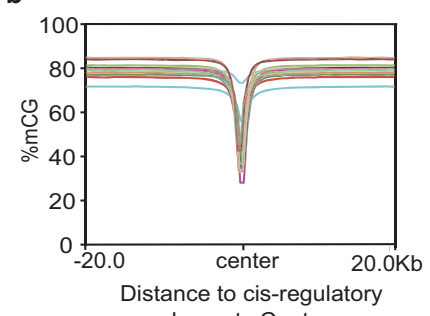

elements Center
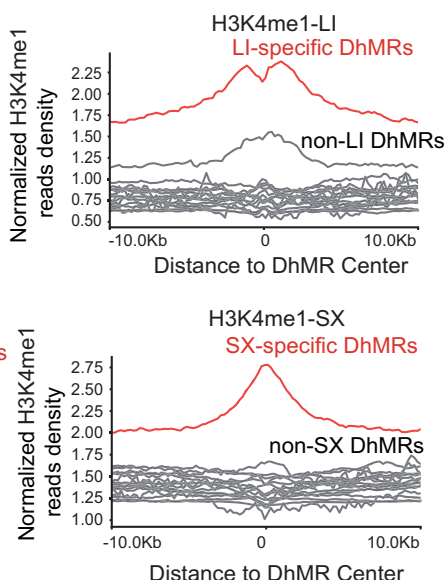

Distance to DhMR Center g

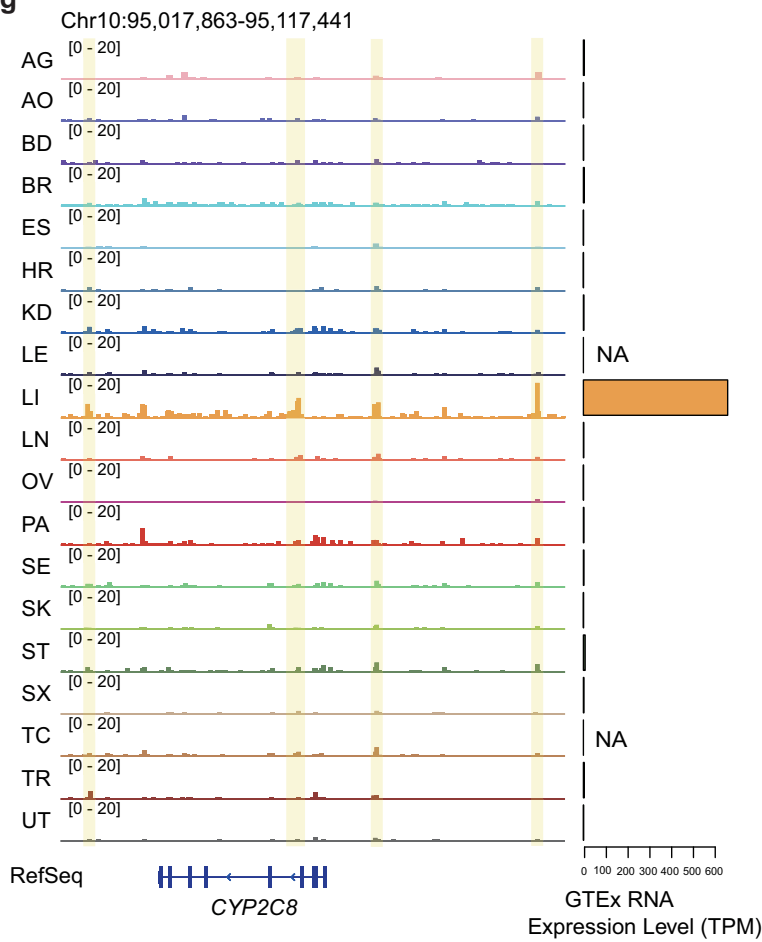

e

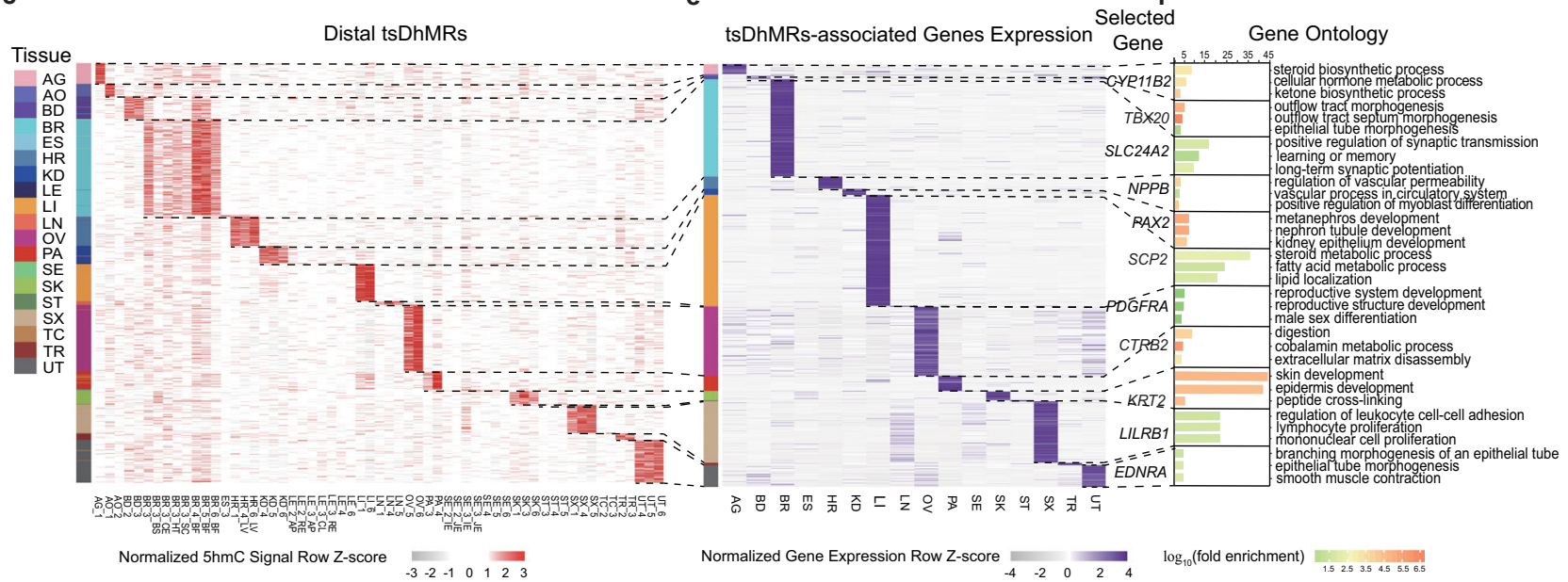

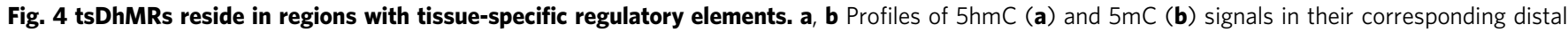
regulatory elements. The distal regulatory elements defined by the ENCODE Project Consortium. c Heatmap showing the normalized 5 hmC signals in distal tsDhMRs. d Profiles of H3K27ac and H3K4me1 modifications around distal tsDhMRs in the liver (LI) and spleen (SX). Red represents the tissue type mentioned in the title of each panel and gray denotes the rest of the tissue types. e Heatmap showing the expression of genes associated with dista tsDhMRs within $500 \mathrm{~kb}$. $\mathbf{f} \mathrm{GO}$ enrichment and representative genes of distal tsDhMR-associated genes. $\mathbf{g}$ IGV visualization of the $5 \mathrm{hmC}$ signals near CYP2C8 on chromosome 10. The highlighted regions are liver-specific DhMRs.

total, we identified 2,279 genes whose expression showed significant correlations with $5 \mathrm{hmC}$ signals in tsDhMRs (Fig. $4 \mathrm{e}$ and Supplementary Fig. 5e). These were genes with tissue-specific expression, such as CYP11B2 in adrenal glands and $N P P B$ in heart. Gene ontology (GO) analysis demonstrated that these genes are enriched in tissue-specific functions, such as learning or memory (brain), kidney epithelium development (kidney), female gonad development (ovary) and sex differentiation (uterus) (Fig. 4f and Supplementary Fig. 5f). Similarly, KEGG pathway analysis revealed that tsDhMR-associated genes participate in tissue-specific functional pathways (Supplementary Fig. $5 \mathrm{~g}$ ). CYP2C8, which is involved in the metabolism of xenobiotics, is highly expressed in liver, with liver-specific DhMRs upstream of CYP2C8 (Fig. 4g). Meanwhile, PTF1A plays a vital role in mammalian pancreatic development; and there are pancreasspecific DhMRs around the gene (Supplementary Fig. 5h). In summary, tsDhMRs may contain putative regulatory elements, which specifically affect the expression of nearby functional genes.

Tissue-specific transcription factors are enriched in tsDhMRs. We next analyzed potential transcription factor-binding site (TFBS) within tsDhMRs. We found that more than half of the tsDhMRs overlapped with at least one TFBS (Supplementary Fig. $6 \mathrm{a})$, and $20.1 \%$ of the distal tsDhMRs even overlapped with 
a

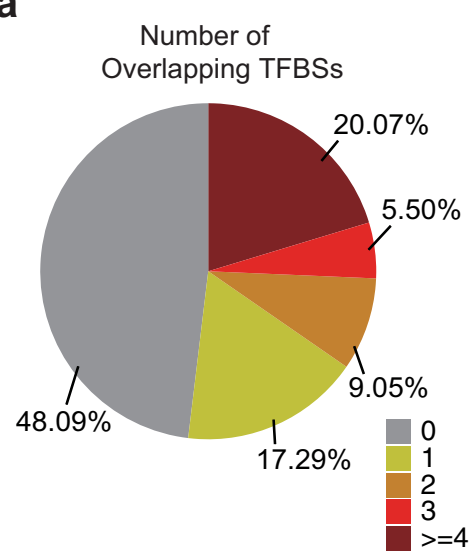

C
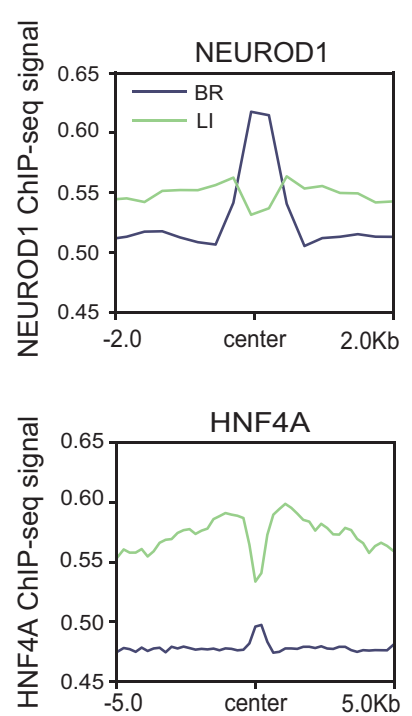

e

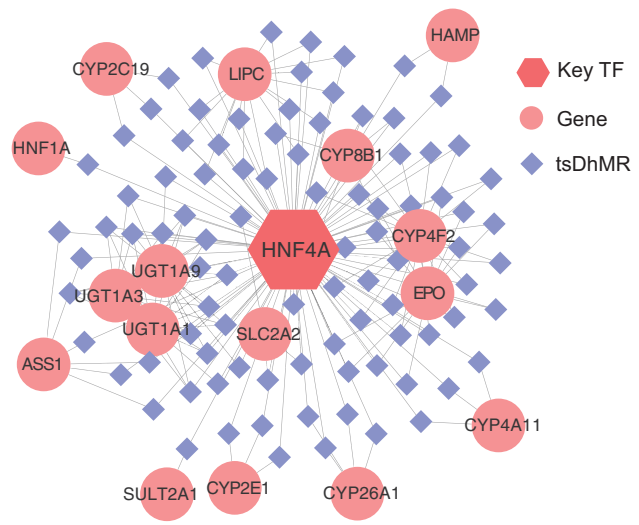

b

d
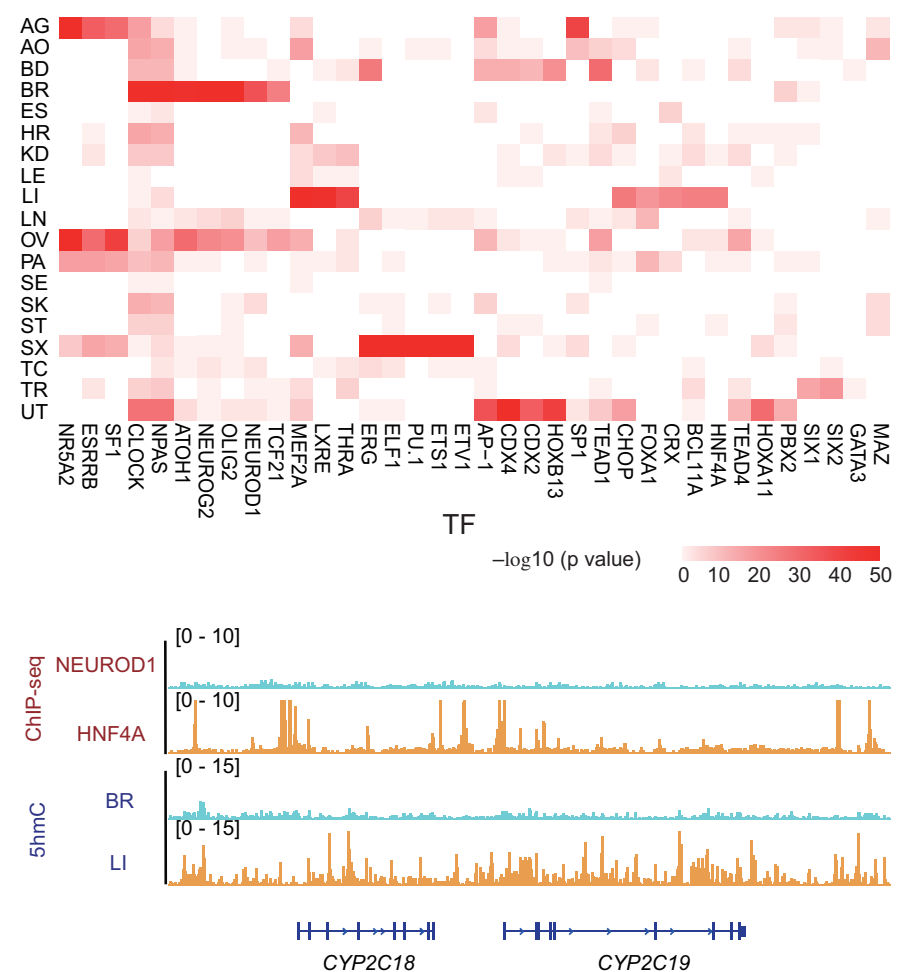

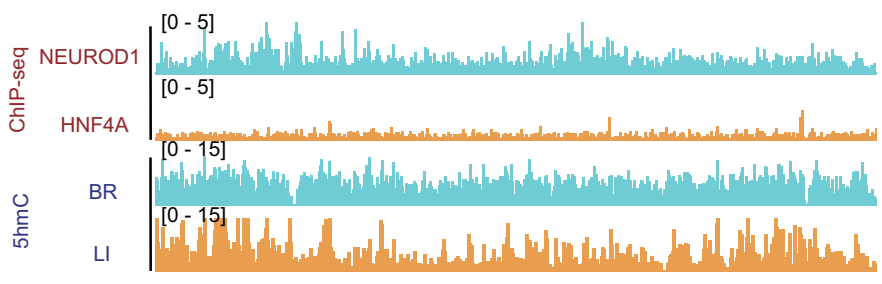

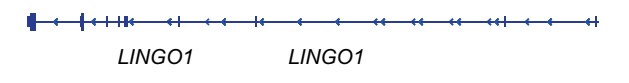

f

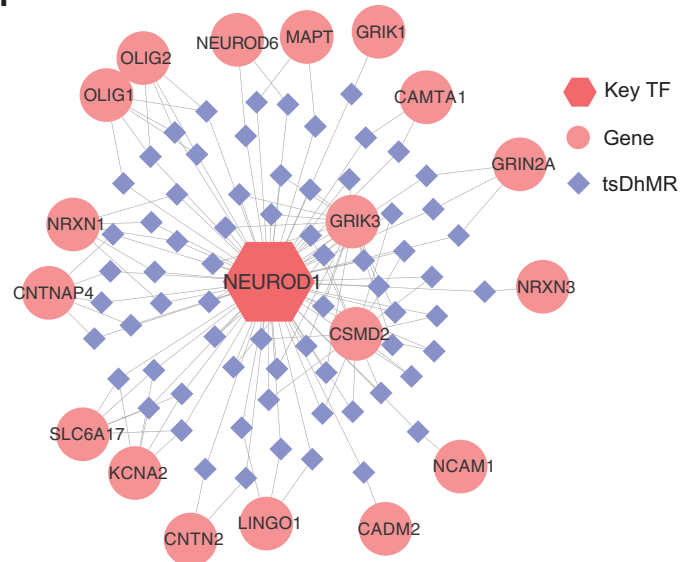

Fig. 5 tsDhMRs are enriched for tissue-specific TFBSs. a Overlap of distal tsDhMRs with ENCODE TFBSs. $\mathbf{b}$ The TF motifs enriched in distal tsDhMRs of each tissue. The color scale represents the - $\log _{10}(P$-value). One-side binomial test (default by homer2). c ChIP-seq signals of NEUROD1 and HNF4A around BR-specific DhMRs and LI-specific DhMRs. d IGV visualization of 5hmC signals and ChIP-seq signals of NEUROD1 and HNF4A in the brain and liver. e, $\mathbf{f}$ Key TF regulatory networks in the liver (e) and brain (f). Hexagon represents key TF; rhombi represents tsDhMRs; circles represents regulated genes. Lines linking the hexagon and rhombi indicate that the TF binds to the tsDhMRs, which is supported by TF ChIP-seq data.

no less than four TFBSs (Fig. 5a). We further investigated different transcription factors (TFs) enriched in tsDhMRs derived from each tissue type and found that tissue-specific TFs were significantly enriched in the corresponding tsDhMRs, regardless of H3K4me1/H3K27ac modification (Fig. 5b and Supplementary Fig. 6b, c). Taking NEUROD1 and HNF4A as examples, NEUROD1 plays an important role in the regulation of the differentiation process of various nervous system cells during 
development, while HNF4A regulates the expression of several hepatic genes. By comparing to ENCODE ChIP-seq data, we found that NEUROD1 specifically binds to brain-specific DhMRs, while HNF4A specifically binds to liver-specific DhMRs (Fig. 5c and Supplementary Fig. 6d). We further used LINGO1 and CYP2C18, which are downstream targets of NEUROD1 and HNF4A in the brain and liver, respectively, to demonstrate the tissue-specific coexistence of $5 \mathrm{hmC}$ signals (Fig. $5 \mathrm{~d}$ ). Thus, key tissue-specific TFs may rewire their tissue-specific network through binding to tsDhMRs.

To further understand the regulatory functions, we constructed regulatory networks mediated by HNF4A and NEUROD1 with their corresponding tsDhMRs. We found that HNF4A can regulate genes with tissue-specific expression, such as CYP4F2, $C Y P 8 B 1$, and UGT1A9, via a process potentially mediated by liver-specific DhMRs (Fig. 5e). Within the NEUROD1 network, brain-specific DhMRs regulate GRIK3, CSMD2 and other genes to perform their brain-specific functions (Fig. 5f). Through network analysis, we illustrated that the key TFs interact with tsDhMRs, which may further affect the expression of tissuespecific functional genes.

GWAS SNPs are preferentially located within tsDhMRs. We next analyzed the potential relationship of tsDhMRs with functional single-nucleotide polymorphisms (SNPs). We found that the tsDhMRs derived from each tissue highly overlapped with the GTEx single-tissue eQTL SNPs (Fig. 6a). In fact, tsDhMRs contain SNPs that are functional in the corresponding tissues. Moreover, tsDhMRs are also enriched for GWAS SNPs ${ }^{34}$ with phenotypes related to the corresponding tissue functions (Fig. 6b and Supplementary Fig. 6e, f), indicating that tsDhMRs reside in regulatory elements that associate with tissue-related diseases. We used several examples to elaborate the findings: (1) SNPs related to electrocardiographic traits and QRS duration were highly enriched in heart-specific DhMRs; (2) SNPs related to metabolite levels and LDL cholesterol were enriched in liver-specific DhMRs; and (3) SNPs related to type 2 diabetes were enriched in pancreas- and adrenal gland-specific DhMRs.

More specifically, we used an example to illustrate the potential mechanism by which distal GWAS SNPs may impact diseases. $H C N 4$, which is necessary for the cardiac pacemaking process, is specifically expressed in the heart (Fig. 6c). We found that several heart-related GWAS SNPs were localized in the heart-specific DhMR (Fig. 6c), indicating that the DhMR is associated with heart diseases. Moreover, several enhancers of the heart identified by ENCODE candidate cis-regulatory elements (cCREs) were located in this DhMR (Fig. 6c). We further integrated the VISTA enhancer data ${ }^{35}$, which were validated by transgenic mouse assays, and found an enhancer (hs2161 from VISTA) within the DhMR. This enhancer is specifically expressed in the mouse heart (images from VISTA database) (Fig. 6d). These data confirm that DhMR, located in enhancer, is functional in the heart and is related to heart diseases. These results indicate that dysregulation of tsDhMRs may be involved in human disease pathologies. Collectively, our data show that tsDhMRs could help us understand the function of distal GWAS SNPs in the corresponding tissues.

\section{Discussion}

In this study, we present a base-resolution atlas of $5 \mathrm{hmC}$ in human tissues. Hundreds of thousands of $5 \mathrm{hmC}$ peaks and millions of $5 \mathrm{hmC}$ sites were identified in this dataset, expanding the epigenomic landscape determined by previous large-scale efforts, for example, the ENCODE project. While our work was under review, a $5 \mathrm{hmC}$ tissue map of adults of European descent was published, which found that $5 \mathrm{hmC}$ is preferentially enriched on tissue-specific gene bodies and enhancers ${ }^{36}$. Thus, tissuespecificity of $5 \mathrm{hmC}$ is validated in different human ancestry. Moreover, this study has enabled additional findings: (1) The correlation between $5 \mathrm{hmC} / 5 \mathrm{mC}$ levels and gene expression in multiple human tissues has not been directly compared previously. We found that tissue-specifically expressed genes show tissue-specific $5 \mathrm{hmC}$ patterns, which is not observed for $5 \mathrm{mC}$; (2) tsDhMRs reside in regions with regulatory potential to control the expression of nearby tissue-specific functional genes; (3) tsDhMRs are enriched with tissue-specific transcription factorbinding sites and rewire the regulatory network of tissue-specific transcription factors; (4) tsDhMRs reside in regulatory elements that associate with tissue-related functional GWAS SNPs.

Our $5 \mathrm{hmC}$ tissue map is base-resolution, owning to the use of hmC-CATCH ${ }^{21}$. This feature not only improves the confidence of $5 \mathrm{hmC}$ detection (Supplementary Fig. 1e), but also reveals information with regard to the detailed distribution pattern of $5 \mathrm{hmC}$ (Fig. 2). For instance, we found a "CAhmCGT" motif that resembles the binding motif of ARNT While approximately half of ARNT binding sites contain $5 \mathrm{hmC}$, only a small part of $5 \mathrm{hmC}$ sites contain ARNT binding sites. Thus, ARNT binding only partially explains the $5 \mathrm{hmC}$ motif. Among the many possibilities for this observation, other transcription factors could be invovled.

Gene body $5 \mathrm{hmC}$ levels are positively correlated with gene expression, especially of genes with tissue-specific expression. Thus, the gene body $5 \mathrm{hmC}$ levels may be used to infer gene expression in tissues. This can be particularly useful in some precious clinical samples, where RNA degradation is severe (frozen samples, formalin-fixed paraffin-embedded samples, body fluids and so on). For instance, $5 \mathrm{hmC}$ levels in cell-free DNA (cfDNA) have been utilized as biomarkers for cancer diagnosis ${ }^{21,37-39}$. While it is anticipated that healthy and cancerous tissues may have different hydroxymethylomes, cancerspecific $5 \mathrm{hmC}$ signatures, which may reflect the gene expression program in different cancers, could be identified for noninvasive cancer diagnosis. Thus, the relative stability of epigenetic modifications makes $5 \mathrm{hmC}$ a promising candidate for prediction of gene expression in clinical samples.

Using the hydroxymethylome of multiple tissues, we discovered that approximately one-third of all $5 \mathrm{hmC}$ peaks are tissue-specifically hyperhydroxymethylated. We found that tsDhMRs, residing in potential regulatory regions, are positively correlated with gene expression, which is in contrast to the fact that differentially methylated regions (DMRs) show a negative correlation $^{40}$. Moreover, tissue-specific TFs are enriched in tsDhMRs, providing a mechanism by which key TFs may regulate tissue-specific gene expression via tsDhMRs. Based on our identified tsDhMRs, future studies could be designed to illustrate the specific mechanisms by which $5 \mathrm{hmC}$ can regulate tissue development and differentiation.

Through integration of GWAS SNP and $5 \mathrm{hmC}$ data, we discovered that tsDhMRs were significantly enriched with GWAS SNPs. Our data indicate that tsDhMRs contribute to tissuerelated diseases. Although GWAS SNPs are consistent among somatic cells, they may affect tsDhMR 5hmC levels and ultimately lead to dysfunctions of the corresponding tissues. Our analysis provides new insights into the understanding of GWAS data, where distal GWAS SNPs interact with tsDhMRs to regulate target genes. Disruption of tsDhMR $5 \mathrm{hmC}$ levels may result in tissue-related disease phenotypes.

Collectively, our data provide a rich resource for understanding the $5 \mathrm{hmC}$ landscape in human tissues. The reported human tissue hydroxymethylome adds to the knowledge of how this epigenetic mark may affect tissue-specific differentiation and diseases. 
a

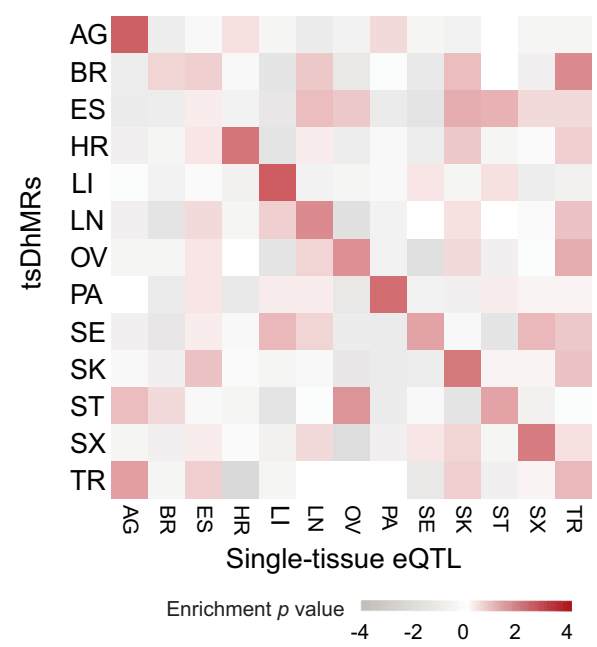

d

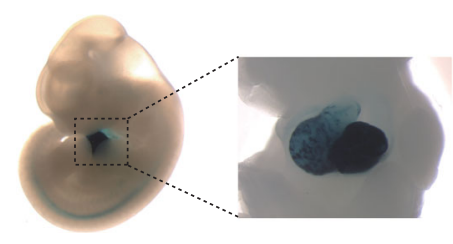

hs2161 b

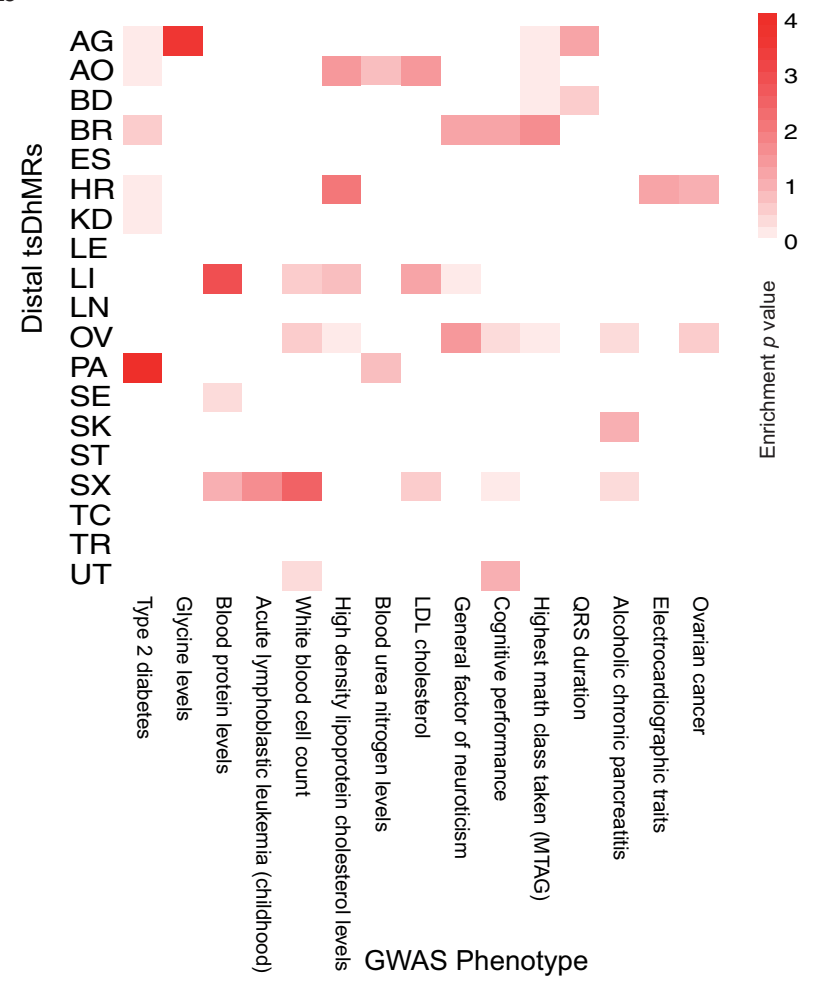

c

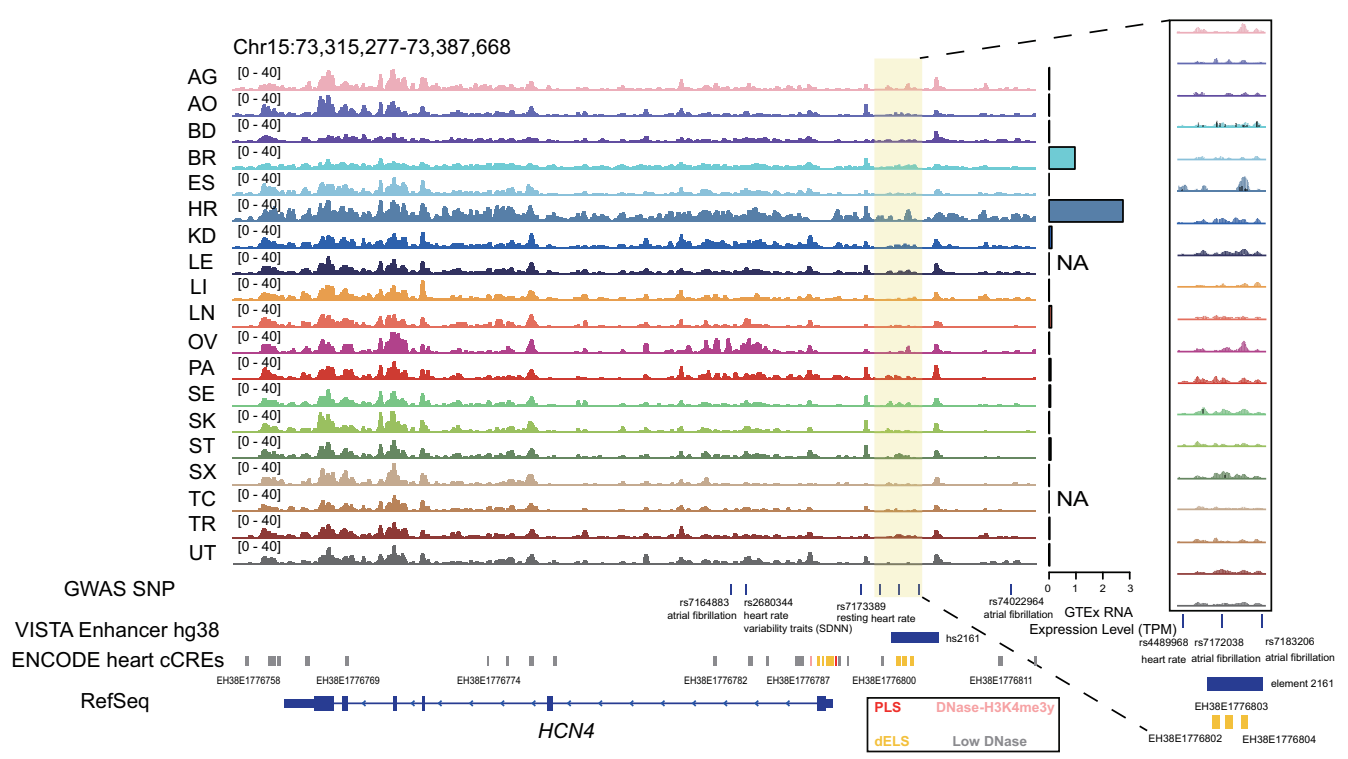

Fig. 6 tsDhMRs enrich tissue-specific, phenotypic GWAS SNPs. a Overlap of tsDhMRs with single-tissue eQTL SNPs. eQTL SNP data are from GTEx. One-sided (greater) fisher's exact test. b Representative GWAS phenotypes enriched in distal tsDhMRs. One-sided (greater) fisher's exact test. c IGV visualization of the $5 \mathrm{hmC}$ signals around HCN4 on chromosome 15. The locations of GWAS SNPs and VISTA enhancers are also shown. The highlighted region shows the LI-specific DhMRs. $\mathbf{d}$ In vivo reporter assay of enhancer activity for hs2162. This pictures were obtained from the VISTA enhancer browser ${ }^{35}$

\section{Methods}

Biospecimen collection. We collected a total of 60 tissue samples from deceased donors who had died of natural and accidental deaths at Zhongshan Hospital (Shanghai, China); we targeted 19 distinct tissues from 6 postmortem Chinese individuals, including 3 males and 3 females. All samples were obtained research consent from the families and informed consent under a protocol approved by the ethics committee of School of Basic Medical Sciences of Fudan University, China. The collected samples were transferred to cryovials for long-term storage at $-80^{\circ} \mathrm{C}$.

Preparation of genomic DNA. Genomic DNA was isolated from tissues using the Blood/Cell/Tissue Genomic DNA Extraction Kit (TIANGEN, DP304) following the manufacturer's specifications. The $5 \mathrm{hmC}$ sequencing libraries were constructed with $200 \mathrm{ng}$ of genomic DNA. Briefly, the DNA was fragmented with a ME220 Focused-ultrasonicator (Covaris, ME220) to 300-500 bp. We added a model sequence as a spike-in to the fragmented DNA (Ref, $5 \mathrm{hmC}$ and $5 \mathrm{fC}$ spike-in, Supplementary Table 3 ) followed by NEBNext ${ }^{\oplus}$ End Repair Module (NEB) according to the manufacturer's protocol. Then, the repaired DNA samples were purified by 1.8x AMPure XP (Beckman Coulter) according to the manufacturer's protocol.

Hydroxylamine-mediated blocking of endogenous 5fC. Repaired DNA was added to $10 \mathrm{mM}$ O-ethylhydroxylamine (Aldrich, 274992) in $50 \mu \mathrm{L} 100 \mathrm{mM}$ MES 
buffer ( $\mathrm{pH} 5.0)$ at $37^{\circ} \mathrm{C}(850 \mathrm{rpm})$ for $4 \mathrm{~h}$ in a thermomixer (Eppendorf). $5 \mathrm{fC}$ blocked DNA was purified with AMPure XP beads (Beckman Coulter) and Micro Bio-Spin P-6 SSC column (Bio-Rad).

$\mathrm{K}_{2} \mathrm{RuO}_{4}$-mediated oxidation of genomic DNA and labeling. The 5fC-blocked DNA was denatured in $0.05 \mathrm{M} \mathrm{NaOH}$ (total volume $48.5 \mu \mathrm{L}$ ) for $30 \mathrm{~min}$ at $37^{\circ} \mathrm{C}$. The reaction was snap cooled on ice-water bath $\left(0^{\circ} \mathrm{C}\right)$ for $5 \mathrm{~min}$. Add $1.5 \mu \mathrm{L} 15 \mu \mathrm{M}$ $\mathrm{K}_{2} \mathrm{RuO}_{4}$ to the denatured DNA sample and briefly mix the reaction. Leave the oxidation reaction on ice for $1 \mathrm{~h}$. Moreover, using a Bio-Rad Micro Bio-Spin P-6 SSC column (Bio-Rad) to purify the oxidized sample. Then, the purified sample was added to $0.3 \mathrm{mg} \mathrm{AI}$ (J\&K scientific, 2793949) in $100 \mu \mathrm{L} 20 \mathrm{mM}$ Tris-HCl buffer (pH 8.0) at $37^{\circ} \mathrm{C}$ for $18-20 \mathrm{~h}$ in a thermomixer (Eppendorf, 850 r.p.m.) and purified by MinElute PCR Purification Kit (QIAGEN).

Library preparation and click chemistry. The AI-labeled ssDNA was used directly for library preparation with the TELP protocol except PCR amplification ${ }^{41}$. Frist, mixing $28 \mu \mathrm{L}$ of AI-labeled ssDNA, $1 \mu \mathrm{L}$ of $10 \times$ EX buffer (Takara), $1 \mu \mathrm{L}$ of $1 \mathrm{mM}$ dCTP (NEB) and $1 \mu \mathrm{L}$ of terminal deoxynucleotidyl transferase (TDT; NEB) for $37^{\circ} \mathrm{C}$ for $35 \mathrm{~min}, 75^{\circ} \mathrm{C}$ for $20 \mathrm{~min}$. Second, the following extension mix to the above-mentioned TDT reaction: $6.2 \mu \mathrm{L}$ of $\mathrm{H}_{2} \mathrm{O} ; 0.8 \mu \mathrm{L}$ of KAPA2G Robust HS (KAPA); $12 \mu \mathrm{L}$ of $5 \times$ KAPA buffer A (KAPA); $4.8 \mu \mathrm{L}$ of $2.5 \mathrm{mM}$ dNTP (Takara) and $6 \mu \mathrm{L}$ extension primer (Ex primer, Supplementary Table 3). The extension program was as follows: (i) $95^{\circ} \mathrm{C}$ for $3 \mathrm{~min}$; (ii) $47^{\circ} \mathrm{C}$ for $1 \mathrm{~min}, 68^{\circ} \mathrm{C}$ for $2 \mathrm{~min}, 16$ cycles and (iii) $72^{\circ} \mathrm{C}$ for $10 \mathrm{~min}$. Moreover, using exonuclease I (Exo I) (NEB) digest excess primers at $37^{\circ} \mathrm{C}$ for $1 \mathrm{~h}$. Then, the extended DNA was purified by MinElute PCR Purification Kit. Thirdly, mixing $8.4 \mu \mathrm{L}$ DNA, $0.6 \mu \mathrm{L}$ adapter containing unique molecular identifier (UMI), $10 \mu \mathrm{L} 2 \mathrm{x}$ quick ligation buffer (NEB) and $1 \mu \mathrm{L}$ Quick T4 ligase (NEB) for $20^{\circ} \mathrm{C} 1 \mathrm{~h}$. Then the reaction was purified with MinElute PCR Purification Kit. Then, the ligated DNA was added to DBCO-S-SPEG3-Biotin (Click Chemistry Tools, Cat. No. A112-10) with the final concentration of $400 \mathrm{mM}$, and incubated in the thermomixer for $1 \mathrm{~h}$ at $37^{\circ} \mathrm{C}(800$ rpm), and purified by DNA Clean \& Concentrator 5 (Vistech).

Enrichment of 5hmC-containing DNA. The Dynabeads MyOne Streptavidin C1 (Invitrogen) was used to enrich the biotin-labeled DNA according to the manufacturer's protocol. Beads were then resuspended and incubated in freshly prepared $50 \mathrm{mM}$ DTT to release the $5 \mathrm{hmC}$-containing strand for $1 \mathrm{~h}$ at $37^{\circ} \mathrm{C}$. Then the supernatant was purified with Micro Bio-Spin P-6 Gel Columns (Bio-Rad) to remove DTT. The enriched DNA was amplified with Q5 ${ }^{\circledR}$ Hot Start High-Fidelity 2X Master Mix (NEB) according to the manufacturer's protocol and purified with $1 \times$ AMPure XP beads.

qPCR to detect the enrichment of $5 \mathrm{hmC}$. The qPCR assay had two replicates with SYBR Premix Ex TaqTM II (TAKARA) according to the manufacturer's instructions. The reaction was performed with Roche LightCycler ${ }^{\circledR} 96$ Instrument. For spike-in sequences and primers, see Supplementary Table 3.

Data processing. Illumina sequencing adapters and low-quality reads were removed from raw sequencing data to obtain clean data. We added a sequencing barcode through the hmC-CATCH protocol to mark PCR-duplicated reads. Then, the PCR-duplicated reads were filtered out, and only one read was retained using an in-house script. The final cleaned reads were mapped to hg38 by Bismark (Version: v0.15.0). To enhance the signal-to-noise ratio, we used only read pairs with more than one C-to-T conversion for further analysis.

\section{Assessing the C-to-T conversion rate of $\mathbf{5} \mathbf{h m C}$ sites. We added a model} sequence as a spike-in before constructing the hmC-CATCH library, of which one $\mathrm{C}$ site was $100 \%$ hydroxymethylated. After treatment through the hmC-CATCH protocol, we observed a C-to-T conversion signal in this $5 \mathrm{hmC}$ site. We used the $T /(C+T)$ of the sequencing data at this site to estimate the $\mathrm{C}$-to- $\mathrm{T}$ conversion rate.

Identification of $\mathbf{5 h m C}$ sites. We used Bismark (Version: v0.15.0) to extract single-base-resolution information. Sites with less than five total bases (NT + NC) or three NTs were discarded for $5 \mathrm{hmC}$ calling. Then, we used the binomial distribution with $\mathrm{N}$ as the sequencing depth $(\mathrm{NC}+\mathrm{NT})$ and $\mathrm{p}$ as the normal cytosine conversion rate to assess the probability of observing NT by chance. We considered $5 \mathrm{hmC}$ sites with the Holm-Bonferroni method-adjusted $P<0.001$ and located within a type of tissue $5 \mathrm{hmC}$-enriched region as high-confidence $5 \mathrm{hmC}$ sites.

Identification of $\mathbf{5 h m C}$ peaks. Our hmC-CATCH approach could enrich DNA fragments with $5 \mathrm{hmC}$. Here, we used a peak calling method to identify these regions with 5hmC. MACS2 (Version: v2.1.1) was applied to call peaks in each sample with the following command:

"macs2 callpeak $-\mathrm{t}<5 \mathrm{hmC}$ bam $>-\mathrm{c}<$ input bam $>$-g hs - f BAMPE-keep-dup all-outdir $<$ outdir $>$-n $<$ sample name $>$ "
Annotation of $\mathbf{5 h m C}$ sites and peaks. The $5 \mathrm{hmC}$ sites or peaks were annotated by annotatePeaks.pl (Homer, Version: v4.5), and the "-annStats" parameter was added to quantify the enrichment of genomic elements compared to the background. Then, the $\log 2$ ratios of observation to expectation in all tissues were plotted as a bar chart by ggplot2 (R package, Version v3.5.1).

tSNE cluster of the global $5 \mathrm{hmC}$ signals. The whole hg38 genome was first cut into $10-\mathrm{kb}$ non-overlapping bins. Then, the read counts in each $10-\mathrm{kb}$ bin of all samples were calculated by "Bedtools multicov" (bedtools, Version: v2.27.1). After normalizing the sequencing depth (DESeq2, R package) and batch effect (limma, R package, Version v3.5.1), we performed tSNE clustering (Rtsne, R package, Version v3.5.1) to reduce the high-dimensional data to two dimensions. ggplot2 (R package, Version v3.5.1) was used to visualize the data.

Analysis of the correlation of gene body $5 \mathrm{hmC}$ and $5 \mathrm{mC}$. The RPKM values of all protein-coding genes were calculated and normalized as mentioned above. Spearman's correlation coefficients between the RPKM values and $5 \mathrm{hmC} / 5 \mathrm{mC}$ signals in matched tissues were calculated by R (cor, method = "spearman", Version v3.5.1).

Identification of genes with tissue-specific expression. We used GTEx gene expression data (RNASeQCv1.1.9_gene_median_tpm) to identify the genes with tissue-specific expression that were defined as being highly expressed in one tissue We first filtered out the genes with a mean TPM $<1$, which was regarded as low expression in all tissues. Then, we calculated the fold changes in the expression of protein-coding genes in one tissue over the mean values for other tissues. We ordered the genes according to fold change levels, and the top 300 in each tissue were regarded as genes with tissue-specific expression. To ensure that the top 300 genes with tissue-specific expression in each tissue were significantly more highly expressed than others, we filtered out the genes with fold changes lower than 2.

Identification of tissue-specific differential $\mathbf{5 h m C}$ regions. We first merged all $5 \mathrm{hmC}$ peaks from the 60 samples to obtain the total peaks using "Bedtools merge" (bedtools, Version: v2.27.1). Then, the read counts in each merged peak of all samples were calculated by "Bedtools multicov" (bedtools, Version: v2.27.1) and normalized as mentioned above. We merged all biological replicates from the same tissue to enhance the signals. We used the Poisson distribution to estimate the $P$ value of each peak in a tissue. The probability of read counts in each peak of one tissue was estimated by the one-tail Poisson distribution with the parameter $\lambda$ as the mean for other tissues. The $P$-values were further adjusted by the Bonferroni method. The peaks with adjusted $P$-values $<0.05$ and fold changes $>2$ were regarded as significant tissue-specific differential $5 \mathrm{hmC}$ regions.

Identification of tsDhMR-associated genes. We adapted a method to link tsDhMRs to putative genes ${ }^{33}$. We first identified all possible genes linked to tsDhMRs by searching any TSS of GENCODE (Version: v28) protein-coding genes within $500 \mathrm{~kb}$ of the tsDhMRs. Then, we calculated the Pearson correlation coefficients between normalized 5hmC signals and gene expression (TPM). To avoid spurious associations, we used cor.test (R, Version v3.5.1) to assess the stochastic links. Finally, we required that the confident links had a Pearson correlation over 0.8 and $P$-value lower than 0.05 .

Motif-enrichment analysis. For each tissue, we used findMotifsGenome.pl (Homer, Version: v4.5) to find the motifs enriched in tsDhMRs with the following command:

"findMotifsGenome.pl <tsDhMRs bed $>$ hg38 < output>-p 5"

Analysis of tsDhMRs associated with GWAS SNPs. We downloaded previously published GWAS datasets from the GWAS catalog 34 . Then, we calculated the associations of GWAS phenotypes and tsDhMRs. A GWAS phenotype always be associated with multiple SNPs. For each phenotype, we used Fisher's test to compute the significance and odds ratios between the phenotype-associated SNPs and tsDhMRs.

Reporting summary. Further information on research design is available in the Nature Research Reporting Summary linked to this article.

\section{Data availability}

The data that support this study are available from the corresponding authors upon reasonable request. Sequencing data have been deposited into the Gene Expression Omnibus (GEO) under the accession number GSE134078. This study used previously published data including BS-seq (GSE119981, GSE112356, GSE59395) ${ }^{42-44}$, ChIP-seq data for histone modifications (GSE59395, GSE16256) ${ }^{44,45}$ and DNase-seq (GSE18927) $^{45}$, RNA-seq data from GTEx (https://gtexportal.org/home/datasets) ${ }^{30}$, and the GWAS datasets from the NHGRI-EBI GWAS catalog (https://www.ebi.ac.uk/gwas/ docs/file-downloads) ${ }^{34}$. All data related to the manuscript have been published. 


\section{Code availability}

The analysis scripts have been deposited into GitHub [https://github.com/herb-BO/ hmC_CATCH].

Received: 30 June 2020; Accepted: 15 June 2021;

Published online: 12 July 2021

\section{References}

1. Greenberg, M. V. C. \& Bourc'his, D. The diverse roles of DNA methylation in mammalian development and disease. Nat. Rev. Mol. Cell Biol. 20, 590-607 (2019).

2. Smith, Z. D. \& Meissner, A. DNA methylation: roles in mammalian development. Nat. Rev. Genet. 14, 204-220 (2013).

3. Kriaucionis, S. \& Heintz, N. The nuclear DNA base 5-hydroxymethylcytosine is present in purkinje neurons and the brain. Science 324, 929-930 (2009).

4. Tahiliani, M. Conversion of 5-methylcytosine to 5-hydroxymethylcytosine in mammalian DNA by MLL partner TET1. Science 324, 930-935 (2009).

5. Ito, S. et al. Tet proteins can convert 5-methylcytosine to 5-formylcytosine and 5-carboxylcytosine. Science 333, 1300-1303 (2011).

6. He, Y. F. et al. Tet-mediated formation of 5-carboxylcytosine and its excision by TDG in mammalian DNA. Science 333, 1303-1307 (2011).

7. Shen, L. et al. Genome-wide analysis reveals TET- and TDG-dependent 5methylcytosine oxidation dynamics. Cell 153, 692-706 (2013).

8. Maiti, A. \& Drohat, A. C. Thymine DNA glycosylase can rapidly excise 5formylcytosine and 5-carboxylcytosine: potential implications for active demethylation of CpG sites. J. Biol. Chem. 286, 35334-35338 (2011).

9. Bachman, M. et al. 5-Hydroxymethylcytosine is a predominantly stable DNA modification. Nat. Chem. 6, 1049-1055 (2014).

10. Ficz, G. et al. Dynamic regulation of 5-hydroxymethylcytosine in mouse ES cells and during differentiation. Nature 473, 398-402 (2011).

11. Song, C. X. et al. Selective chemical labeling reveals the genome-wide distribution of 5-hydroxymethylcytosine. Nat. Biotechnol. 29, 68-72 (2011).

12. Schutsky, E. K. et al. Nondestructive, base-resolution sequencing of 5hydroxymethylcytosine using a DNA deaminase. Nat. Biotechnol. 36, 1083-1090 (2018).

13. Song, C. X. et al. Sensitive and specific single-molecule sequencing of 5hydroxymethylcytosine. Nat. Methods 9, 75-77 (2011).

14. Haffner, M. C. et al. Global 5-hydroxymethylcytosine content is significantly reduced in tissue stem/progenitor cell compartments and in human cancers. Oncotarget 2, 627-637 (2011).

15. Pfeifer, G. P., Xiong, W., Hahn, M. A. \& Jin, S. G. The role of 5hydroxymethylcytosine in human cancer. Cell Tissue Res. 356, 631-641 (2014).

16. Ficz, G. \& Gribben, J. G. Loss of 5-hydroxymethylcytosine in cancer: cause or consequence? Genomics 104, 352-357 (2014).

17. Lian, C. G. et al. Loss of 5-hydroxymethylcytosine is an epigenetic hallmark of melanoma. Cell 150, 1135-1146 (2012).

18. Mellen, $\mathrm{M}$. et al. MeCP2 binds to $5 \mathrm{hmC}$ enriched within active genes and accessible chromatin in the nervous system. Cell 151, 1417-1430 (2012).

19. Zeng, H., He, B. \& Yi, C. Compilation of modern technologies to map genomewide cytosine modifications in DNA. Chembiochem 20, 1898-1905 (2019).

20. Wu, H. \& Zhang, Y. Charting oxidized methylcytosines at base resolution. Nat. Struct. Mol. Biol. 22, 656-661 (2015).

21. Zeng, H. et al. Bisulfite-free, nanoscale analysis of 5-hydroxymethylcytosine at single base resolution. J. Am. Chem. Soc. 140, 13190-13194 (2018).

22. Zeng, H. et al. Unnatural cytosine bases recognized as thymines by DNA polymerases by the formation of the watson-crick geometry. Angew. Chem. Int Ed. Engl. 58, 130-133 (2019).

23. Zhu, C. et al. Single-cell 5-formylcytosine landscapes of mammalian early embryos and ESCs at single-base resolution. Cell Stem Cell 20, 720-731.e725 (2017).

24. Xia, B. et al. Bisulfite-free, base-resolution analysis of 5-formylcytosine at the genome scale. Nat. Methods 12, 1047-1050 (2015).

25. $\mathrm{Yu}, \mathrm{M}$. et al. Base-resolution analysis of 5-hydroxymethylcytosine in the mammalian genome. Cell 149, 1368-1380 (2012).

26. Lister, R. et al. Human DNA methylomes at base resolution show widespread epigenomic differences. Nature 462, 315-322 (2009).

27. Lister, R. et al. Global epigenomic reconfiguration during mammalian brain development. Science 341, 1237905 (2013).

28. Tian, X. et al. Circulating tumor DNA 5-hydroxymethylcytosine as a novel diagnostic biomarker for esophageal cancer. Cell Res. 28, 597-600 (2018).

29. Jjingo, D. et al. On the presence and role of human gene-body DNA methylation. Oncotarget 3, 462-474 (2012).
30. Consortium, G. T. The genotype-tissue expression (GTEx) project. Nat. Genet. 45, 580-585 (2013).

31. Han, D. et al. A highly sensitive and robust method for genome-wide $5 \mathrm{hmC}$ Profiling of rare cell populations. Mol. Cell 63, 711-719 (2016).

32. Wang, L. et al. Programming and inheritance of parental DNA methylomes in mammals. Cell 157, 979-991 (2014).

33. Corces, M. R. et al. The chromatin accessibility landscape of primary human cancers. Science 362, eaav1898 (2018).

34. Buniello, A. et al. The NHGRI-EBI GWAS Catalog of published genome-wide association studies, targeted arrays and summary statistics 2019. Nucleic Acids Res. 47, D1005-D1012 (2019).

35. Visel, A., Minovitsky, S., Dubchak, I. \& Pennacchio, L. A. VISTA Enhancer Browser-a database of tissue-specific human enhancers. Nucleic Acids Res. 35 , D88-D92 (2007).

36. Cui, X. L. et al. A human tissue map of 5-hydroxymethylcytosines exhibits tissue specificity through gene and enhancer modulation. Nat. Commun. 11, $6161(2020)$

37. Song, C. X. et al. 5-Hydroxymethylcytosine signatures in cell-free DNA provide information about tumor types and stages. Cell Res. 27, 1231-1242 (2017).

38. Mellen, M., Ayata, P. \& Heintz, N. 5-hydroxymethylcytosine accumulation in postmitotic neurons results in functional demethylation of expressed genes. Proc. Natl Acad. Sci. USA 114, E7812-E7821 (2017).

39. Li, W. et al. 5-Hydroxymethylcytosine signatures in circulating cell-free DNA as diagnostic biomarkers for human cancers. Cell Res. 27, 1243-1257 (2017)

40. Schultz, M. D. et al. Human body epigenome maps reveal noncanonical DNA methylation variation. Nature 523, 212-216 (2015).

41. Peng, X. et al. TELP, a sensitive and versatile library construction method for next-generation sequencing. Nucleic Acids Res. 43, e35 (2015).

42. Vogel Ciernia, A. et al. Epigenomic convergence of neural-immune risk factors in neurodevelopmental disorder cortex. Cereb. Cortex 30, 640-655 (2020).

43. Blake, L. E. et al. A comparison of gene expression and DNA methylation patterns across tissues and species. Genome Res. 30, 250-262 (2020).

44. Heidari, N. et al. Genome-wide map of regulatory interactions in the human genome. Genome Res. 24, 1905-1917 (2014).

45. Bernstein, B. E. et al. The NIH roadmap epigenomics mapping consortium. Nat. Biotechnol. 28, 1045-1048 (2010).

\section{Acknowledgements}

The authors would like to thank Jiabin Cai and Jia Fan for providing tissue samples; Xushen Xiong for bioinformatics assistance; and National Center for Protein Sciences at Peking University in Beijing, China, for assistance with quantitative PCR analysis. Part of the analysis was performed on the High Performance Computing Platform of the Center for Life Sciences. This work was supported by National Key R\&D Program (no. 2019YFA0110900 to C.Y. and no. 2020YFC2002900 to W.T.), the National Natural Science Foundation of China (nos. 91953201 and 21825701 to C.Y. and nos. 82072826 to Q.Z.), and Peking University Ge Li and Ning Zhao Education Fund.

\section{Author contributions}

C.Y., W.T., and Q.Z. designed the study. 5fC labeling chemical compounds were synthesized by B.D. X.Z., B.H., Y.F., H.Z., and L.J. performed experiments. C.Z. and B.H. analyzed the high-throughput sequencing data with help from H.M., B.H., C.Z., P.J., X.Z., W.T., and C.Y. wrote the manuscript.

\section{Competing interests}

B.X., A.Z., C.Z., and C.Y. are co-inventors on filed patents (PCT/CN2014/087479 and $201710111600.9)$ for the labeling strategies of $5 \mathrm{fC}$ and $5 \mathrm{hmC}$. The other authors declare no competing interests.

\section{Additional information}

Supplementary information The online version contains supplementary material available at https://doi.org/10.1038/s41467-021-24425-w.

Correspondence and requests for materials should be addressed to Q.Z., W.T. or C.Y.

Peer review information Nature Communications thanks Lu Wen and the other, anonymous, reviewer(s) for their contribution to the peer review of this work.

Reprints and permission information is available at http://www.nature.com/reprints

Publisher's note Springer Nature remains neutral with regard to jurisdictional claims in published maps and institutional affiliations. 
(c) (i) Open Access This article is licensed under a Creative Commons Attribution 4.0 International License, which permits use, sharing, adaptation, distribution and reproduction in any medium or format, as long as you give appropriate credit to the original author(s) and the source, provide a link to the Creative Commons license, and indicate if changes were made. The images or other third party material in this article are included in the article's Creative Commons license, unless indicated otherwise in a credit line to the material. If material is not included in the article's Creative Commons license and your intended use is not permitted by statutory regulation or exceeds the permitted use, you will need to obtain permission directly from the copyright holder. To view a copy of this license, visit http://creativecommons.org/ licenses/by/4.0/.

(C) The Author(s) 2021 